\title{
Rank-Based Routing under Blind Information for Cognitive Radio Ad Hoc Networks
}

\author{
Priya Thomas ${ }^{1}$, Subbaroyan $\mathrm{V}^{2}$ \\ ${ }^{1}$ Department of Electronics and Communication, KCG College of Technology, Karapakkam, Chennai, Tamil Nadu-600097, India \\ ${ }^{2}$ Professor, Department of Electronics and Communication, KCG College of Technology, Karapakkam, Chennai, Tamil Nadu-600097, India
}

\begin{abstract}
The concept of opportunistic dynamic spectrum access or the cognitive radio emerged as a result of tremendous increase in the demand for wireless connectivity. In wireless networks, control information is usually carried through a common channel. But in a cognitive radio ad hoc network, secondary users can use only the channels that are not used by primary users. Therefore it is not possible to have a common channel for all nodes in the network. Also, multiple broadcasting of control messages may cause channel congestion and packet collisions. Routing packets without considering budget constraints degrades the overall network performance. Users may not receive the expected Quality of Service (QoS). They may also suffer from heavy monetary loss. In order to tackle the above problems, this paper introduces a server known as the Secondary Service Provider (SSP) with a rank-based routing algorithm. It stores and coordinates control information from different nodes. It optimally distributes the traffic over the spectrum bands based on budget constraints. The system is matched to practical scenarios by the inclusion of blind information. Simulation results prove that throughput and packet delivery fraction have increased considerably well with the use of SSPs. It has also reduced the end-to-end delay.
\end{abstract}

Keywords: Blind information, cognitive radio ad hoc networks, control information, rank-based routing, secondary service provider.

\section{Introduction}

Every application has a specific bandwidth. Due to this fixed spectrum-allocation policy, a major share of the assigned spectrum is underutilized. Cognitive Radios (CR) are a solution to this problem by enabling unlicensed users to exploit the spectrum that is unused by licensed users. The licensed users are called the primary users (PU) and the unlicensed users are called the secondary users (SU) of that spectrum. In a cognitive radio network, every user is free to use all frequencies depending on their availability. There is no restriction on the set of frequencies that can be used. A CR can intelligently detect whether any portion of the spectrum is in use and can temporarily use it without interfering with the transmission of other users [12].

In a dynamic environment like cognitive radio, it is very important that the control information reach every node in the network. Routing information and channel availability information are collectively called as the control information. Hence, proper reception of it is necessary for the secondary users to adaptively adjust their operating parameters. In traditional wireless networks, control information was broadcasted through a common channel. But in cognitive radio networks, the notion of a common channel is not feasible. This is because users acquire different channel sets in different time slots. They do not share a common channel.

In this paper, we introduce a server - the Secondary Service Provider (SSP) that stores control information from every node in its range. These servers are also capable of communicating with each other and thus integrate the control information of all nodes in the network. This mitigates all the broadcast problems that were discussed earlier. Moreover, the system is considered under blind information - absence of the following information: i) Network topology information; ii) Channel availability information; iii) Time synchronization information.

This paper also formulates a ranking scheme for use within SSPs. This helps CR sessions to select appropriate channels for packet delivery. The SSP ranks the channels based on certain parameters. When a service request comes, it chooses the best channel depending on the ranks assigned. For example, when a request for video streaming arrives, the server chooses the channel with the highest bandwidth among all the channels and assigns it to serve the request. The paper simulates the rank-based selection of channels in the cognitive mode of data transmission and the ad hoc involvement in it using Network Simulator (NS2). Figure 1 shows the cognitive radio network.

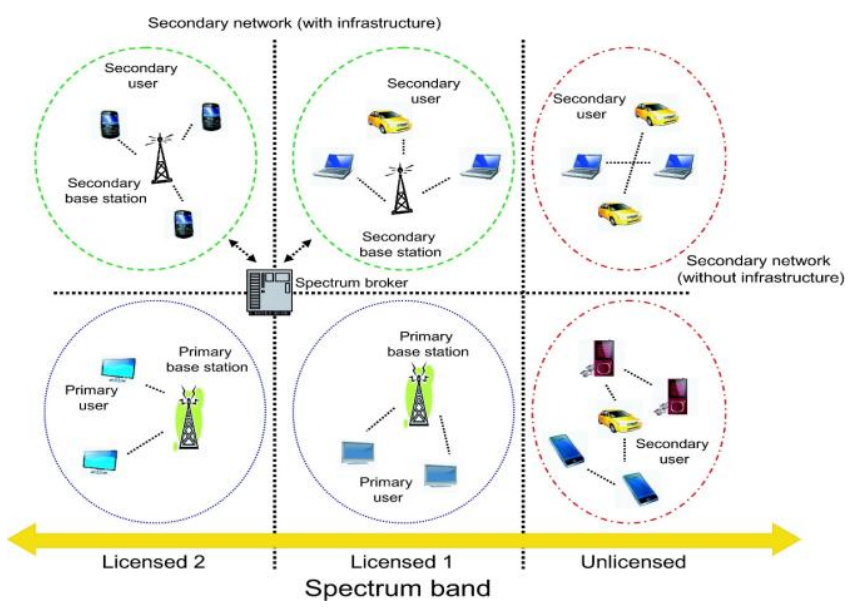

Figure 1: Cognitive radio network in spectrum

Rest of this paper is organized as follows. Section 2 describes previous works related to the proposed concept. We describe the rank-based routing and control in SSPs in section 3. Section 4 elaborates on the software modules used in the work. In section 5, we provide experimental results and 


\section{International Journal of Science and Research (IJSR) \\ ISSN (Online): 2319-7064 \\ Index Copernicus Value (2013): 6.14 | Impact Factor (2014): 5.611}

analysis based on the simulation. Finally, we conclude in section 6 .

\section{Related Work}

In [11], the authors propose a QoS-Based Broadcast Protocol under Blind Information for Multi hop Cognitive Radio Ad Hoc Networks. The protocol designs channel-hopping sequences for both the SU sender and the SU receiver based on random selection of channels. An SU sender randomly broadcasts on a set of channels from its available channels. Broadcasting is repeated on the selected set for multiple time slots to increase the probability of reception.

Pros and cons:

- This scheme increases the success rate of reception of control messages.

- It causes delay in delivery as the broadcast has to be repeated periodically on the randomly selected channels.

In [12], the authors propose a distributed broadcast protocol in multi-hop cognitive radio ad hoc networks with collision avoidance. There are three components for the proposed protocol: 1) construction of broadcasting sequences; 2) distributed broadcast scheduling scheme and 3) broadcast collision avoidance scheme. A time-slotted approach is adopted for SUs where the length of a time slot is sufficient to transmit a broadcast packet.

Pros and cons:

- It provides a high successful delivery ratio and short broadcast delay.

- SUs are assumed to be static. Also, each SU is assumed to know the locations of its all two-hop neighbors.

In [6], the authors address the issue of dynamic assignment of control channel in cognitive radio networks based on time and space varying spectrum environment. They propose a cluster-based architecture that allocates different control channels at various clusters in the network. The problem is formulated as a bipartite graph problem.

Pros and cons:

- It facilitates for graceful channel migration when primary radio activity is detected, without the need for frequent reclustering.

- Significant computational overhead is involved in the formation of dynamic clusters.

In [9], the authors present two channel-hopping-based MAC layer protocols, SYNC-ETCH and ASYN-ETCH, for communication rendezvous in CR networks. SYNC-ETCH assumes that $\mathrm{CR}$ nodes can synchronize their channel hopping processes. ASYNC-ETCH does not rely on global clock synchronization.

Pros and cons:

- The protocols achieve communication rendezvous in CR networks.

- It is not fully applicable to practical scenarios as it considers time synchronization.
In [3], the authors provide a survey on the routing metrics used in CR networks. They list the challenges that have to be addressed in designing a good routing metric. They provide taxonomy of different metrics and a survey on how they are used in different routing protocols. The paper also compares different classes of metrics and provides ways to combine individual routing metrics to obtain a global one.

In [5], the authors propose a Dynamic Multilevel Priority packet scheduling scheme. Each node has three levels of priority queues. Real-time packets are placed into the highest-priority queue and non-real-time packets are placed into two other queues based on a certain threshold of their estimated processing time. It outperforms conventional schemes in terms of average data waiting time and end-to-end delay.

\section{Rank-Based Routing in SSPs}

As we have already seen, every node in a network broadcast control information periodically. Many protocols have been designed to increase the probability that every other node in a network receive control messages from every other node. But they cannot be better than having a controller that can collect control messages from all the nodes and perform necessary information exchange with the nodes. In this paper, we introduce a server called as the Secondary Service Provider.

The SSPs reside within Mobile Switching Centres (MSCs) of the network. An SSP stores the control information sent by a node within its range. These SSPs can cover all the nodes in a network by installing sufficient number of them in the network. These servers then communicate with each other to integrate the status information of all nodes present in the network. Depending on the stored information in SSPs, MSC takes decision on the call transfers whenever a service request comes.

Besides this, SSPs use a ranking mechanism to route the packets belonging to different applications. In this, every channel is allocated with a set of three parameters - cost, bandwidth and security. The SSP ranks the channels based on these parameters. When a service request comes, it chooses the best channel among the available channels depending on the ranks assigned. For example, when a voice call arrives, the server chooses the channel with the least cost among all the channels and assigns it to serve the call.

\section{Software Modules}

The system implementation is carried out in four modules. They are:

1. Application selection

2. Initializing parameters

3. Channel selection

4. Selection algorithm

The block diagram of the proposed system is as shown below. 


\section{International Journal of Science and Research (IJSR) \\ ISSN (Online): 2319-7064}

Index Copernicus Value (2013): 6.14 | Impact Factor (2014): 5.611

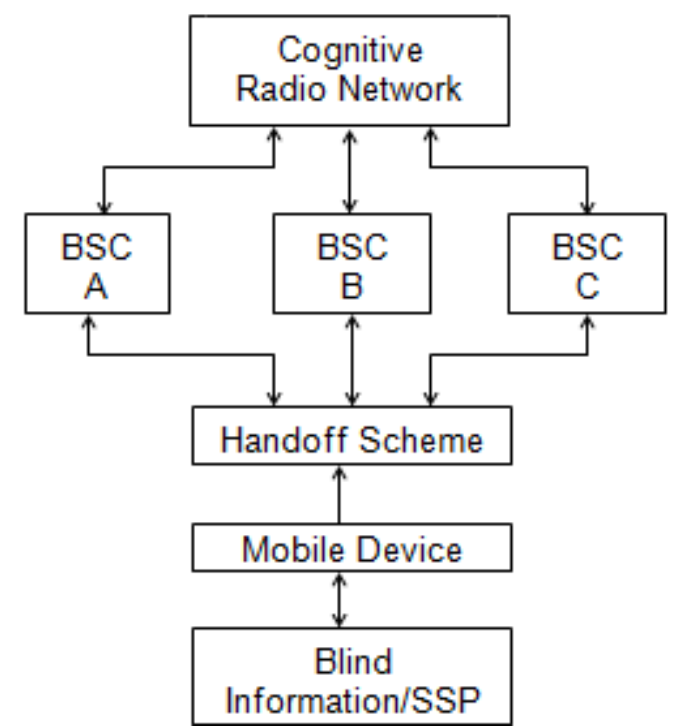

Figure 2: Block diagram of the proposed system

\subsection{Application Selection}

This is the user interactive module. The user selects the application of his choice. The applications considered in this system are voice, video and e-Transaction. Each application is considered to have specific user preferences as shown in table 1 .

Table 1: User preferences

\begin{tabular}{|c|c|c|c|}
\hline Application & Bandwidth & Cost & Security \\
\hline Voice & Medium & Low & Low \\
\hline Video & High & Medium & Low \\
\hline e-Transaction & Medium & Low & High \\
\hline
\end{tabular}

\subsection{Initializing Parameters}

Every channel is allocated with a set of three parametersbandwidth, cost and security. In this module, each of these parameters is initialized for all the available channels. The parameters are assigned 1, 0 or -1 depending on their weight.

- 1 represents High

- 0 represents Medium

- -1 represents Low

These are the normalized values of parameters.

\subsection{Channel Selection}

Here, we compare the parameter values of the available channels. Based on this comparison, a rank list is generated for every parameter. Now, SSP gives suggestion on the best channel to the node that requested the service. If the application selected is voice, then the channel with the least cost of service is chosen to serve the request. If the application is video, then the channel having the highest bandwidth is chosen. If the application is e-Transaction, then the channel with the most secure connection is found out.

\subsection{Selection Algorithm}

This module can be considered as the backbone of the system. It involves the discovery and selection mechanism to find a new base station. The proposed path selection algorithm works on the choice of user specified QoS parameters.

Algorithm:

Step 1: Start

Step 2: Customize channel parameters.

Step 3: Store values of channel parameters.

Step 4: Prompt user to input the application.

Step 5: Compare and sort the corresponding parameter of all the channels.

Step 6: Generate a rank list for the parameter.

Step 7: Select the appropriate channel based on the rank list.

- If application=voice, then choose the channel with the least cost of service.

- Else if application=video, then choose the channel having the highest bandwidth.

- Else (application=e-Transaction) choose the channel which is the most secure.

Step 8: End

\section{Results and Analysis}

A total of 16 nodes are simulated. Out of these, node- 0 is the MSC. Nodes-1, 2, 3 are the Base Station Controllers (BSCs). Nodes-4, 5, 6, 7, 8, 9, 10 are the substations. Nodes-11, 12, 13,14 are the mobile nodes. Nodes-15 and 16 represent the SSPs. Each BSC handles a specific band of frequencies. The colors-green, yellow and blue are used to represent the three different frequency sets used by the BSCs. Figure 3 shows the network topology.

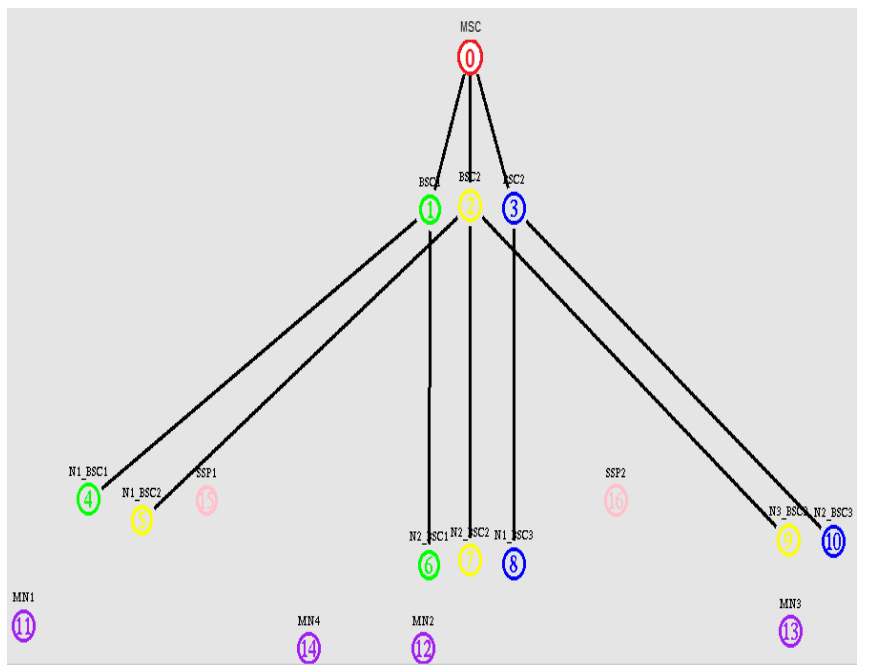

Figure 3: Network topology

\subsection{Selection of Application}

Node-11 is configured as the sender node and node-13 as the receiver node. User will be prompted to enter his choice of application in the terminal window.

\subsection{Selection of Channels}

The entire test area is divided into three regions: R1, R2 and R3.

- R1: Sender node (node-11) is under the coverage of substations 4 and 5. 


\section{International Journal of Science and Research (IJSR) \\ ISSN (Online): 2319-7064 \\ Index Copernicus Value (2013): 6.14 | Impact Factor (2014): 5.611}

- R2: Node-11 is under the coverage of sub-stations 6, 7 and 8.

- R3: Node-11 is under the coverage of sub-stations 9 and 10.

Initially, node-11 and node-13 are in R1 and R3 respectively. Node-11 is moving towards R3 and node-13 is moving towards R1. Let the parameter values for the three channels are as shown in table 2 .

Table 2: Channel parameter values

\begin{tabular}{|c|c|c|c|}
\hline Channel & Bandwidth & Cost & Security \\
\hline 1 & 1 & 0 & -1 \\
\hline 2 & 0 & -1 & 1 \\
\hline 3 & -1 & 1 & 0 \\
\hline
\end{tabular}

According to the above table,

- Channel-1 has the highest bandwidth.

- Channel-2 has the least cost.

- Channel-2 provides the highest security.

The appropriate channels will be selected for packet delivery based on the application chosen. Also, the above channel parameter values can be customized in the program.

\subsection{Graphical Analysis}

Output graphs as shown below are generated after successful simulation of the system in NS2. It gives an estimation of the performance of the existing and proposed systems, communication with simple server SSPs as well as through SSPs incorporated with ranking mechanism. Evaluation is done for throughput, end-to-end delay and packet delivery fraction. The output graphs given below correspond to the application-voice.

\subsubsection{Throughput}

It is defined as the total number of packets delivered over the total simulation time. The graph in figure 4 shows that for a definite number of nodes, the proposed system gives better throughput than the existing system.

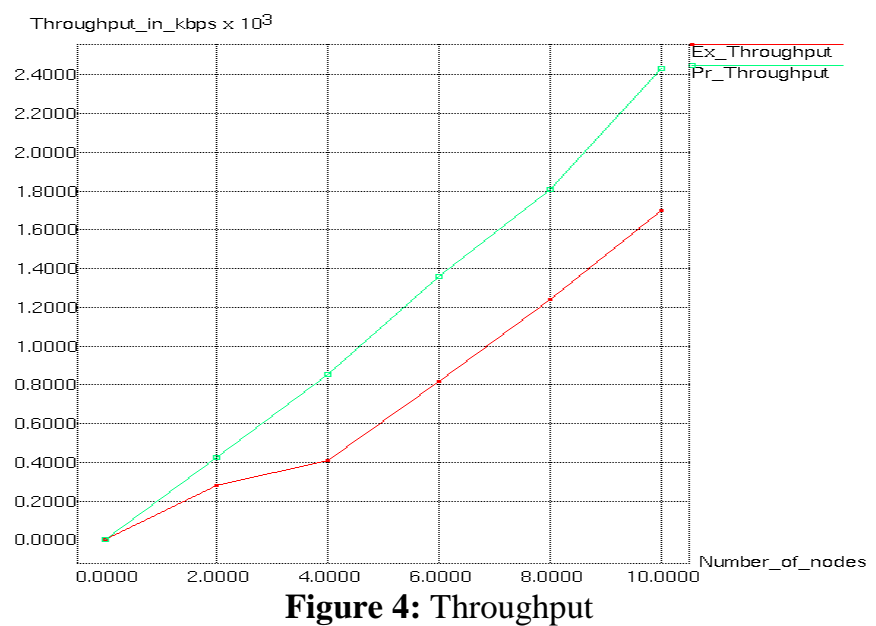

\subsubsection{End-to-End Delay}

It is the average time a data packet takes to reach the destination. This metric is calculated by subtracting the time at which first packet was transmitted by the source from the time at which it arrived at the destination. As seen in figure 5, the end-to-end delay has reduced considerably in the proposed system.

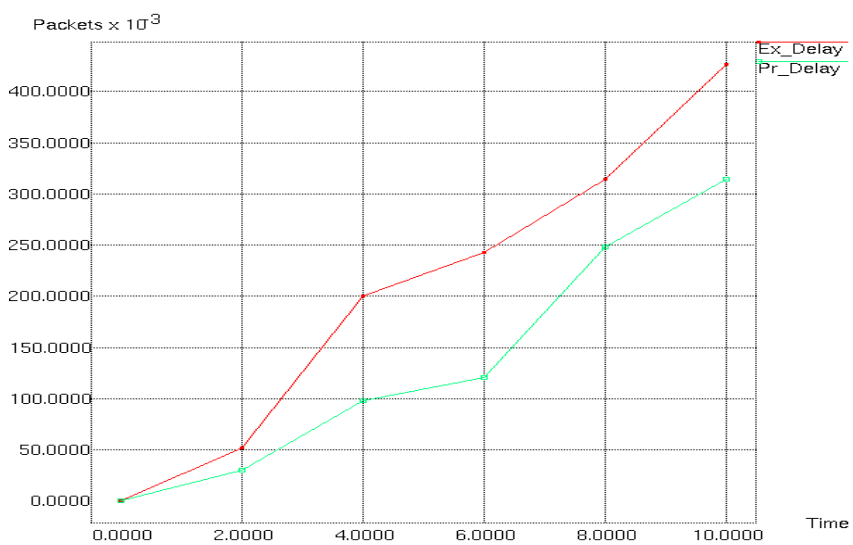

Figure 5: End-to-End Delay

\subsubsection{Packet Delivery Fraction}

It is the ratio of data packets delivered to the destination to those generated at the source. This is more for the new system.

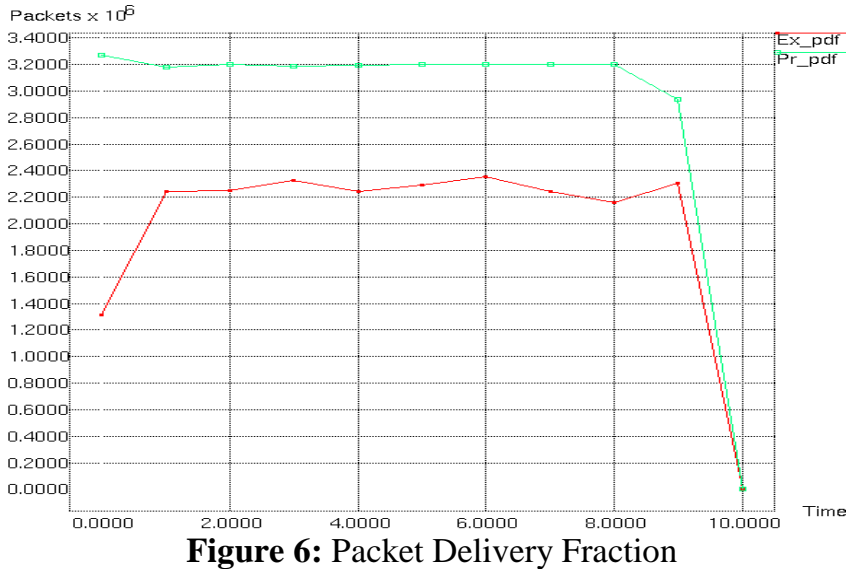

\section{Conclusion}

In this paper, we have used a server-the secondary service provider to solve the broadcast issues in multi hop cognitive radio ad hoc networks. Software reference models for the existing and proposed systems are designed and implemented in NS2. The rank-based routing algorithm in SSPs improves spectrum utilization in cognitive radios. The system is made applicable to practical scenarios with the inclusion of blind information.

The proposed system increases the overall throughput and the packet delivery fraction of the system. There is greater probability that all nodes in a network successfully receive the control information. It resolves the problem of broadcast delay, thereby reducing the average end-to-end delay. Broadcast collisions can also be mitigated. It considers budget constraints in path selection. It reduces the delay in servicing requests. It provides a cost effective means of communication. 


\section{References}

[1] Cunhao Gao, Yi Shi, Y. Thomas Hou, Hanif D. Sherali and Huaibei Zhou, "Multicast Communications in MultiHop Cognitive Radio Networks", IEEE Journal on selected areas in Communications, vol. 29, no. 4, 2011.

[2] Kaigui Bian, Jung-Min Park and Ruiliang Chen, "Control Channel Establishment in Cognitive Radio Networks using Channel Hopping", IEEE Journal on selected areas in Communications, vol. 29, no. 4, 2011.

[3] Moustafa Youssef, Mohamed Ibrahim, Mohamed Abdelatif, Lin Chen, and Athanasios V. Vasilakos, "Routing Metrics of Cognitive Radio Networks: A Survey", IEEE Communications Surveys \& Tutorials, vol. 16, no. 1, 2014.

[4] Nick C. Theis, Ryan W. Thomas and Luiz A. DaSilva, "Rendezvous for Cognitive Radios", IEEE Transactions on Mobile Computing, vol. 10, no. 2, 2011.

[5] Nidal Nasser, Lutful Karim and Tarik Taleb, "Dynamic Multilevel Priority Packet Scheduling Scheme for Wireless Sensor Network", IEEE Transactions on Wireless Communications, vol. 12, no. 4, 2013.

[6] Sisi Liu, Loukas Lazos and Marwan Krunz, "Clusterbased Control Channel Allocation in Opportunistic Cognitive Radio Networks", IEEE Transactions on Mobile Computing, vol. 11, no. 10, 2012.

[7] Stergios Stotas and Arumugam Nallanathan, "Enhancing the Capacity of Spectrum Sharing Cognitive Radio Networks", IEEE Transactions on Vehicular Technology, vol. 60, no. 8, 2011.

[8] Tigang Jiang, Honggang Wang and Athanasios V. Vasilakos, "QoE-Driven Channel Allocation Schemes for Multimedia Transmission of Priority-Based Secondary Users over Cognitive Radio Networks", IEEE Journal on selected areas in Communications, vol. 30, no. 7, 2012.

[9] Yifan Zhang, Gexin Yu, Qun Li, Haodong Wang, Xiaojun Zhu and Baosheng Wang, "Channel-HoppingBased Communication Rendezvous in Cognitive Radio Networks", IEEE/ACM Transactions on Networking, vol. 22, no. 3, 2014.

[10]Yi Song and Jiang Xie, "ProSpect: A Proactive Spectrum Handoff Framework for Cognitive Radio Ad Hoc Networks without Common Control Channel", IEEE Transactions on Mobile Computing, vol. 11, no. 7, 2012.

[11] Yi Song and Jiang Xie, "QB²IC: QoS-Based Broadcast Protocol under Blind Information for Multi hop Cognitive Radio Ad Hoc Networks", IEEE Transactions on Vehicular Technology, vol. 63, no. 3, 2014.

[12] Yi Song and Jiang Xie, "BRACER: A Distributed Broadcast Protocol in Multi-Hop Cognitive Radio Ad Hoc Networks with Collision Avoidance", IEEE Transactions on Mobile Computing, vol. 14, no. 3, 2015. 